\title{
Method for Direct Measurement of Structural Rolling Resistance for Heavy Vehicles
}

Nielsen, Natasja R.; Chatti, Karim; Nielsen, Christoffer Peder; Zaabar, Imen; Hjorth, Poul G.; Hecksher, Tina

Published in:

Transportation Research Record

Link to article, DOI:

10.1177/0361198120915699

Publication date:

2020

Document Version

Publisher's PDF, also known as Version of record

Link back to DTU Orbit

Citation (APA):

Nielsen, N. R., Chatti, K., Nielsen, C. P., Zaabar, I., Hjorth, P. G., \& Hecksher, T. (2020). Method for Direct Measurement of Structural Rolling Resistance for Heavy Vehicles. Transportation Research Record, 2674(5), 371-380. https://doi.org/10.1177/0361198120915699

\section{General rights}

Copyright and moral rights for the publications made accessible in the public portal are retained by the authors and/or other copyright owners and it is a condition of accessing publications that users recognise and abide by the legal requirements associated with these rights.

- Users may download and print one copy of any publication from the public portal for the purpose of private study or research.

- You may not further distribute the material or use it for any profit-making activity or commercial gain

- You may freely distribute the URL identifying the publication in the public portal 


\title{
Method for Direct Measurement of Structural Rolling Resistance for Heavy Vehicles
}

Transportation Research Record 2020, Vol. 2674(5) 37I-380

(C) National Academy of Sciences: Transportation Research Board 2020 Article reuse guidelines: sagepub.com/journals-permissions DOI: 10.1 I 77/036 | I 98।209/5699 journals.sagepub.com/home/trr (S)AGE

\author{
Natasja R. Nielsen', Karim Chatti ${ }^{2}$, Christoffer P. Nielsen ${ }^{3}$, Imen Zaabar ${ }^{4}$, \\ Poul G. Hjorth ${ }^{5}$, and Tina Hecksher
}

\begin{abstract}
In this paper, a new in situ method for determining the structural rolling resistance (SRR), defined as the dissipated energy caused by deformation of the pavement when subjected to a moving load, is presented. The method is based on the relation between SRR and the slope of the deflection basin under a moving load. Using the Traffic Speed Deflectometer, the deflection slope is measured at several positions behind and in front of the right rear-end tire pair of a full-size truck trailer while driving under realistic conditions. The deflection slope directly under the tire is estimated from a linear interpolation between the two nearest sensors. A set of data from a test road segment located in Denmark is analyzed and the SRR coefficients are found to be in the range $\mathbf{0 . 0 0 5 \%}$ to $0.05 \%$. The deflection slope measurements have a high reproducibility (repeated measurements agree within standard deviations of $4 \%$ to $10 \%$ ) with high spatial resolution, and the method for calculating SRR from these measurements has the clear advantage that it requires no knowledge or model of the pavement structure or viscoelastic properties. Numerical simulations of pavement response show that the proposed interpolation method tends to underestimate the actual SRR, and better estimates can be obtained by other interpolation schemes.
\end{abstract}

When driving at constant speed, the fuel consumption goes into overcoming driving resistance. Many different factors contribute to the driving resistance in a vehicle; among the most prominent are uphill driving, air drag, internal friction, and rolling resistance (1). It is estimated that for heavy trucks, $15 \%$ to $30 \%$ of the fossil fuel input is used to overcome the rolling resistance (2). Rolling resistance losses arise from two main sources: 1) viscoelastic effects in the tires and 2) effects of the pavement, including unevenness, texture, and viscoelastic deformation of the pavement $(3-5)$. The focus in this paper is on the latter.

An elastic or viscoelastic pavement subject to a moving vehicle will deform underneath the tires. If the pavement is viscoelastic, this deformation will result in energy dissipating into the pavement structure. The lost energy has to be compensated through additional work from the vehicle engine, to maintain a constant driving speed (6). The amount of additional energy needed depends on the structure of the pavement and this will be referred to as structural rolling resistance (SRR) throughout the paper.

The deflection basin under a moving tire $(\mathrm{z}(\mathrm{x}))$ is asymmetric because of the viscoelastic properties of the pavement causing a time delay in the deflection of a viscoelastic pavement. This time delay makes the maximum deflection appear behind the center of the tire, as seen on Figure $1 a$. This means that the tire always will be on an uphill slope $\left(\frac{\partial z(x=0)}{\partial x}>0\right.$ ), (see Figure $1 b$ ) and thus has to do work in order to maintain a constant driving speed (7). Using this uphill slope notion, the SRR can be calculated directly from the asymmetric deflection basin $(1,8,9)$. Deflection of a structure subject to a moving load has been reported in the literature since the 1960s; for example, in (7), the viscoelastic response of a Kelvin beam is analyzed, and the viscoelastic effects reported to manifest themselves through an asymmetric deflection basin.

\footnotetext{
'Department of Science and Environment, Roskilde University, Roskilde, Denmark

${ }^{2}$ Department of Civil and Environmental Engineering, Michigan State University, East Lansing, MI

${ }^{3}$ Greenwood Engineering A/S, Brøndby, Denmark

${ }^{4}$ Department of Computer Science and Engineering, Michigan State University, East Lansing, MI

${ }^{5}$ DTU Compute, Technical University of Denmark, Lyngby, Denmark
}

Corresponding Author:

Natasja R. Nielsen: narini@ruc.dk 

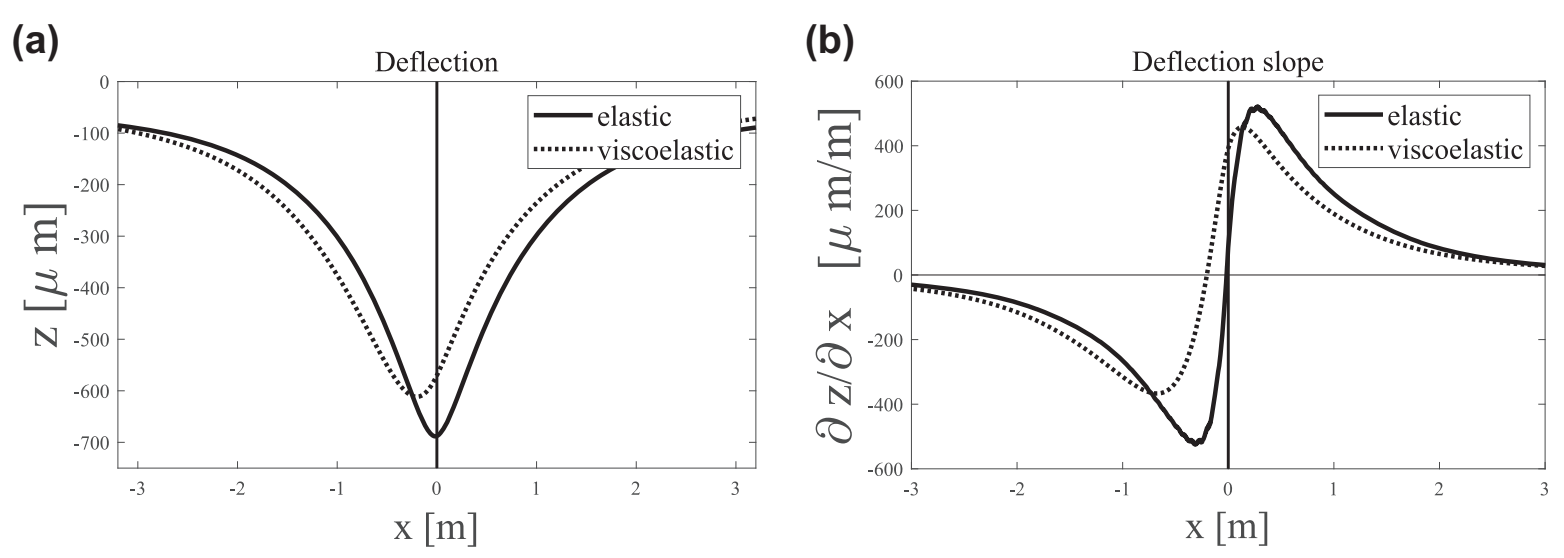

Figure I. (a) Simulated deflection basin underneath a moving load for an elastic (solid line) and viscoelastic (dotted line) pavement, and (b) associated deflection slope for the elastic and viscoelastic pavement. The basin is obtained using a numerical simulation explained at a later point in this paper.

Although SRR has been studied for decades, it has proven difficult to devise accurate and robust ways of measuring it (10). As a consequence, little is known about the absolute magnitude of SRR or its relative contribution to the overall rolling resistance. Indirect measurements of the influence of the dissipative effects in bituminous layers have been estimated by comparing fuel consumption measurements on flexible and rigid pavements. These studies rely on the assumption that rigid pavements have little or no viscous losses and thus the difference in fuel consumption between these types of pavements can be ascribed to the viscous behavior of the asphalt $(8,11,12)$. However, it can be difficult to isolate the effects that relate to the pavement structure from other effects caused by, for example, texture or unevenness (9). In addition, unlike texture and unevenness, the effect from pavement structure is found to be highly dependent on external parameters such as temperature, pavement conditions, and so forth (13). It is therefore difficult to say anything conclusive on SRR influence on fuel consumption based on these types of experiments.

Direct estimates of SRR typically come from simulations of pavement deflections with pavement parameters obtained either from backcalculations using falling weight deflectometer tests or other rheological measurements of the bituminous layer. An often used method is to simulate the pavement response in a finite pavement section, as a moving load is passing with constant speed (14). From the response, one can obtain the displacement field of the pavement surface and calculate the dissipated energy in the pavement $(3,10,12,15,16)$. On the basis of such calculations, it is believed that the SRR loss is smaller than the energy loss caused by pavement texture and unevenness (15), but whether it is negligible or significant enough that it should be included in pavement planning is not clear.

Development of methods for reliable measurement of the pavement's influence on the vehicle fuel consumption is thus highly desirable when making lifecycle assessment studies of pavements and should be included in the development of sustainable pavement designs $(3,6)$.

This paper presents a novel method for determining the SRR under realistic driving conditions using the Traffic Speed Deflectometer technology developed by Greenwood Engineering. The technique measures the slope of the deflection basin between the right pair of rear-end tires of a full-size truck trailer, as it moves at realistic driving speeds. Thus, the uphill slope seen by the tire, which is caused by the deformation of the pavement, is directly measured and, from this, the associated SRR loss can be calculated. The estimated SRR is thus obtained under conditions directly comparable to what normal traffic experiences. The method gives spatially resolved (10-m resolution), reproducible, and robust estimates of SRR, even in road segments where the value fluctuates considerably, making it a reliable and modelfree method to measure SRR.

\section{Aim}

The aim of the paper is to present a new concept for measuring SRR using Traffic Speed Deflectometer (TSD) technology. The TSD measures the slope of the deflection basin under the tires of a truck trailer during driving. The concept and its robustness are demonstrated by pilot measurements of a test road segment of $9 \mathrm{~km}$, and the underlying assumptions are discussed in the light of numerical pavement simulations. 


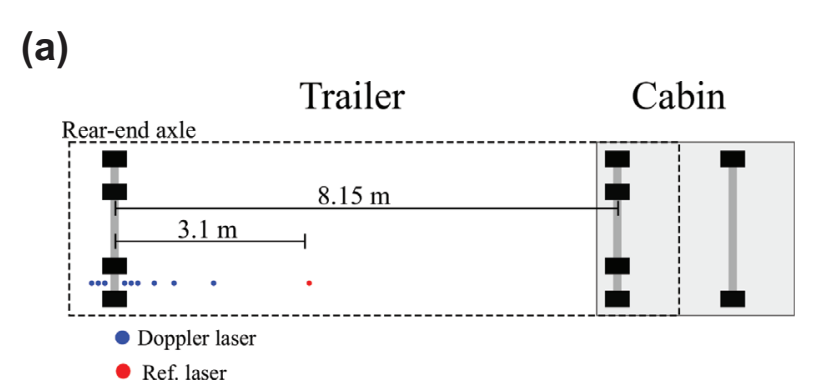

(b)

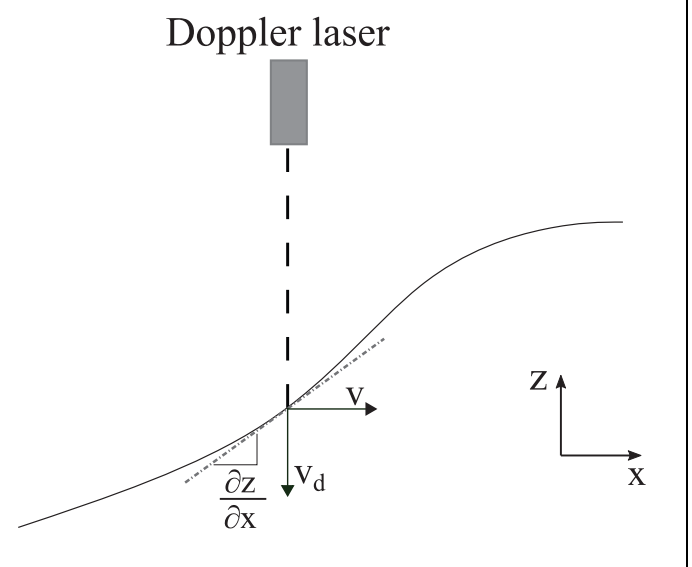

Figure 2. (a) Top view sketch of the Traffic Speed Deflectometer. Nine Doppler lasers are located in between the right rear-end tire pair, as indicated with blue dots. Note that the drawing is not to scale, and the tires in the tire-pair are only separated by $64 \mathrm{~mm}$. (b) Vertical pavement velocity $\left(v_{d}\right)$ at a given point measured using a Doppler laser. See text for further details.

\section{The TSD Concept}

The TSD is conventionally used for continuous bearing capacity measurements by evaluating the slope of the pavement deflection basin. It has the advantage that it makes continuous measurements of the deflection slope and that the TSD trailer is a normal truck trailer and thus can measure under normal driving speed and load as well as measuring directly in the wheel path. In this study a full axle load of 10 tonnes was used.

The TSD device measures the deflection velocity of the pavement as it is subjected to a moving load. This is done by use of Doppler lasers that measure the vertical velocity of the pavement (see Figure $2 b$ ). The TSD truck is equipped with nine Doppler lasers (sensors): three sensors located behind and six in front of the rear-end axle, as shown in Figure $2 a$. Their exact positions relative to the center of the axle (in meters) are

Sensor position $=[-0.366,-0.269,-0.167$, $0.163,0.260,0.362,0.662,0.964,1.559]$.
The measured pavement velocity is adjusted such that effects caused by vertical movements of the truck are subtracted. This is done by using a reference laser mounted $3.1 \mathrm{~m}$ from the rear-end axle, where the deflection of the pavement is assumed zero (red sensor on Figure 2a). The technique is explained in more detail in $(17-20)$.

Figure $2 b$ shows how the vertical pavement velocity $\left(v_{d}\right)$ is measured in a given point using a Doppler laser. The deflection slope at that point $\left(\frac{\partial z}{\partial x}\right)$ corresponds to the slope of the tangent going through the point (gray dotted line) and can be found by dividing $v_{d}$ by the horizontal driving speed $(v)$,

$$
\frac{\partial z}{\partial x}=\frac{\frac{\partial z}{\partial t}}{\frac{\partial x}{\partial t}}=\frac{v_{d}}{v}
$$

The driving speed, $v$, is measured using an odometer located behind the right rear-end tire pair.

\section{Deflection Slope Data}

For this study, three repeated measurements were made with the TSD, on a $9.7-\mathrm{km}$ road section near Copenhagen, Denmark. The measurements were conducted in the spring of 2018 with almost constant air temperature $\left(\sim 14^{\circ} \mathrm{C}\right)$ and road temperature $\left(\sim 18^{\circ} \mathrm{C}\right)$ throughout all three measurements. The driving speed was between $50 \mathrm{~km} / \mathrm{h}$ and $60 \mathrm{~km} / \mathrm{h}$; the exact driving speed was recorded continuously during all measurements. The measured deflection slopes for each sensor were collected at a sampling frequency of 1,000 samples per second and subsequently averaged over $10 \mathrm{~m}$. A plot of the mean value for the three subsequent measurements of each sensor as a function of the driven distance is seen on Figure 3. The measured deflection slope for each sensor varies significantly throughout the measured distance. This variation is however highly reproducible, with average standard deviations between $12 \mu \mathrm{m} / \mathrm{m}$ and $26 \mu \mathrm{m} / \mathrm{m}$ (corresponding to $4-10 \%$ ) between the three measurement runs.

The inset in Figure 3 shows the measured deflection slope as a function of the sensor position measured at $4.5 \mathrm{~km}$ (marked in gray in the main image). The center of the axle in this plot is at $x=0$, indicated with a black dotted line. As mentioned in the introduction, the deflection slope curve is characterized by the minimum deflection slope occurring behind and the maximum deflection slope in front of the tire. The asymmetry in minimum and maximum peak magnitudes is believed to be caused by damping in the pavement. Thus, the location and magnitude of the maximum and minimum carries information about the viscoelastic properties of the pavement.

For analysis of the data, it is necessary to estimate the deflection slope at the axle location, that is, around 


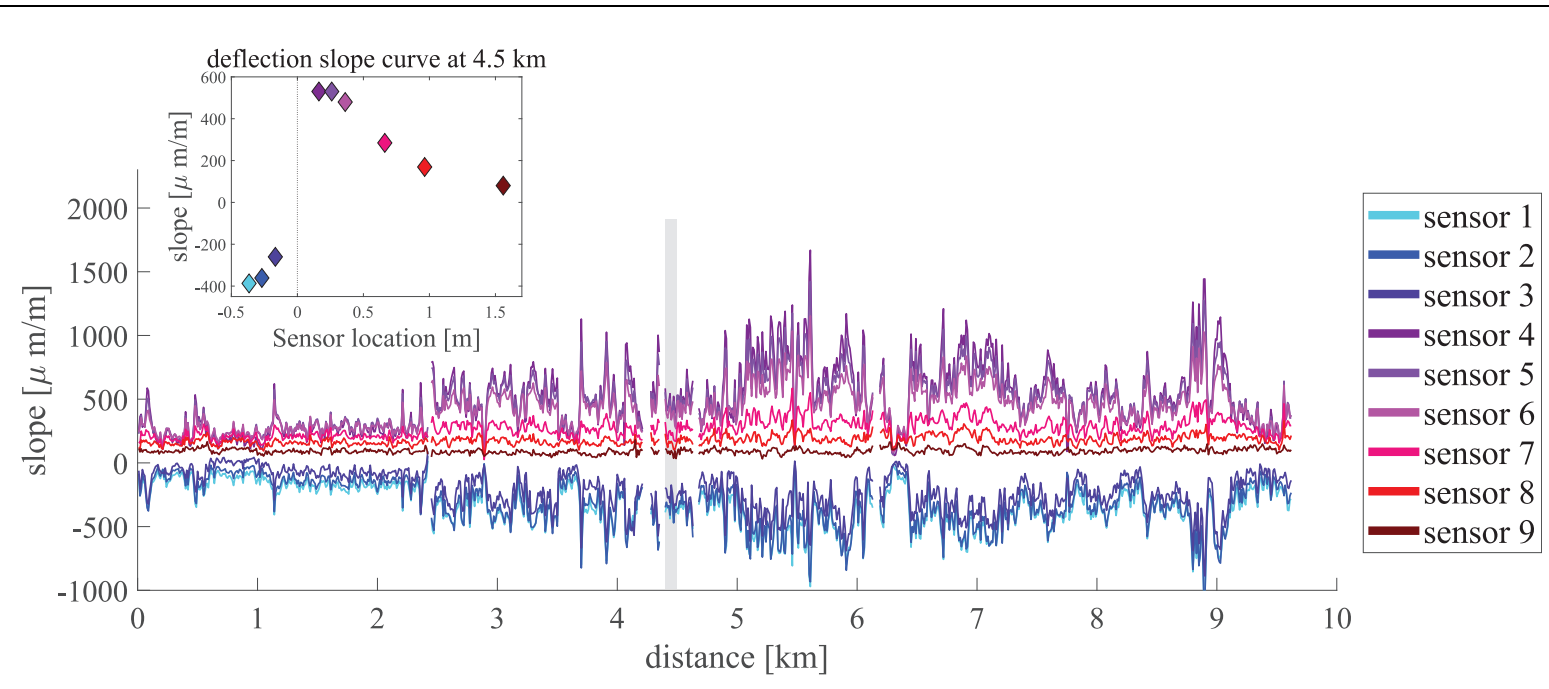

Figure 3. Measured deflection slope for each sensor as a function of the distance, with inset showing a plot of the deflection slopes measured at $4.5 \mathrm{~km}$ as a function of sensor location.

Table I. Partitioning of the Traffic Speed Deflectometer Measurements into Groups

\begin{tabular}{lll}
\hline Group & \multicolumn{1}{c}{ Behavior of signal in Sensors 4, 5, and 6 } & \multicolumn{1}{c}{ Location of maximum } \\
\hline Group 1 & Monotonic decrease & Closer to center of axle than Sensor 4 \\
Group 2 & Increasing or equal from Sensors 4 to 5 and then decreasing in Sensor 6 & $\begin{array}{l}\text { Partly captured by Sensors } 4 \text { and } 5 \\
\text { Froup 3 }\end{array}$ \\
\hline
\end{tabular}

Note: The division is made based on the behavior of the measured deflection slope in Sensors 4, 5, and 6. In total this gives three groups, illustrated in Figure $4 a$.

$x=0$, where a measurement cannot be taken because of the presence of the axle. Instead, the slope must be inferred from the measured locations in front of and behind the center position. This task is easier when the features of the deflection slope are fully captured by the sensors, which is not the case for all traces. Accordingly, the measurements were partitioned into three groups based on the behavior of the signal in Sensors 4, 5, and 6 (Table 1), which gives an indication of where the maximum is located: Group 1 was used for measurements for which the maximum was not captured by the sensors and therefore had to be located closer to the center of the axle than Sensor 4; Group 2 was used for measurements for which the maximum was partly captured by the sensors; and Group 3 was used for measurements for which the maximum was fully captured by the sensors (see Table 1). Examples of measurements from each group are shown in Figure $4 a$. Here the symbols are the average values of the three repeated measurements and the errorbars represent the standard deviations, showing a high degree of reproducibility. Within Groups 1 and 2, a big variation was found in the magnitude of the maximum and the minimum, whereas for measurements belonging to Group 3 this variation was not observed.

\section{Calculating the SRR}

This section shows how the SRR loss can be calculated directly from the measured deflection slope data. In the following it is assumed that the applied load is a point load at the center of the tire, corresponding to $x=0$ and with the magnitude $F_{L}$. The dissipated power caused by $\mathrm{SRR}, P_{\mathrm{SRR}}$, can be found from the applied load and the pavement velocity at this point,

$$
P_{\mathrm{SRR}}=F_{L} v_{d}(x=0)=F_{L} v \frac{\partial z}{\partial x}(x=0)
$$

where the last equality sign comes from Equation 2.

In the case of a perfectly elastic pavement, the maximum deflection will occur directly under the load, making the deflection slope at this point zero and thus $P_{\mathrm{SRR}}=0$. For a viscoelastic pavement, however, the maximum deflection occurs behind the load and there is an uphill slope underneath the load, thus $P_{\mathrm{SRR}}>0$, as 
(a)

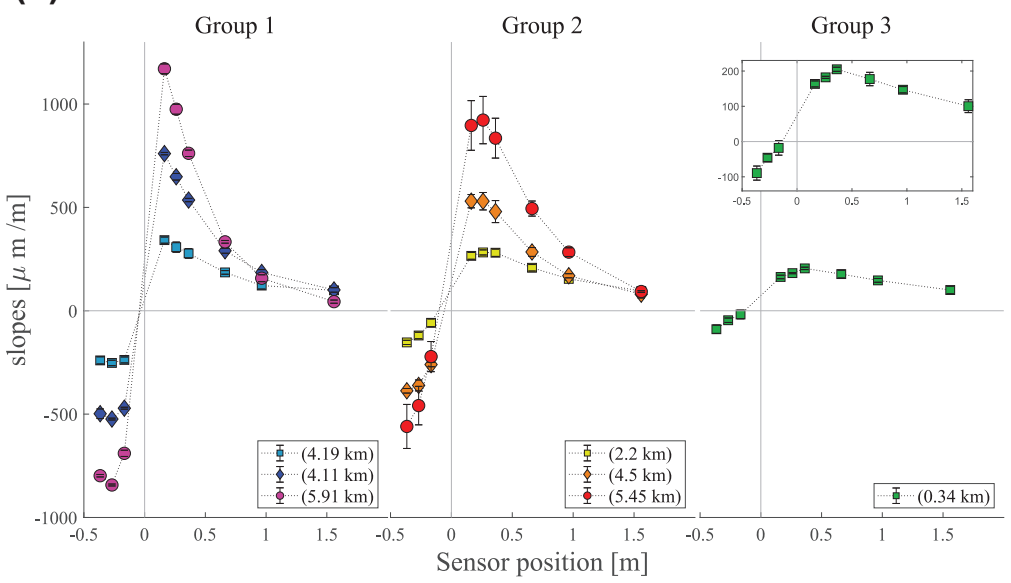

(b)

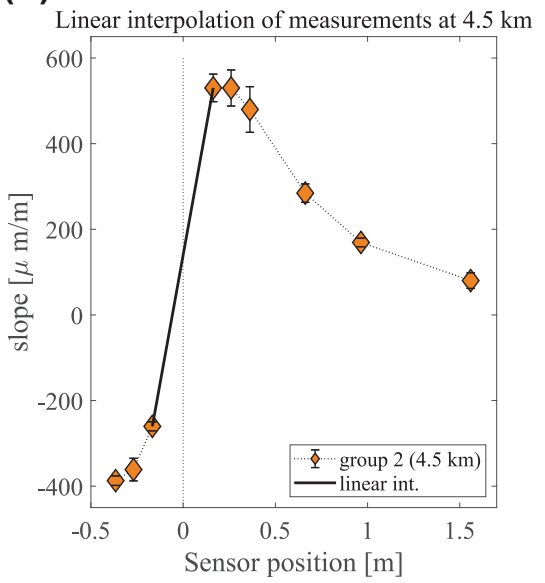

Figure 4. (a) Representative examples of deflection slope plotted as a function of sensor location for the different measurement groups (see Table I), and (b) linear interpolation between the measured values in the two sensors closest to the axle for data measured at $4.5 \mathrm{~km}$ belonging to Group 2.

already illustrated in Figure 1. Note that, the deflection maximum occurs behind the center of the load, whether a point load or a finite contact area is considered. Thus, the tire also experiences an uphill slope if considered a finite contact area, and thereby has $P_{\mathrm{SRR}}>0$ whenever there is damping in the pavement.

To estimate the deflection slope directly under the tire, a linear interpolation is used between the measured deflection slope in the two sensors located closest to the center (Sensors 3 and 4), located at $x=-0.167 \mathrm{~m}$ and $x=0.163 \mathrm{~m}$ respectively, as shown in Figure $4 b$. Therefore the dissipated energy can be written as

$$
P_{\mathrm{SRR}}=F_{L} v b
$$

where $b$ is the intersection of the linear interpolation $\frac{\partial z}{\partial x}(x)=a x+b$ with the $z$-axis, $\frac{\partial z}{\partial x}(x=0)$. From the dissipated power, the rolling resistance force can be defined as $F_{\mathrm{SRR}}=\frac{P_{\mathrm{srr}}}{v}=F_{L} b$. Using the standard definition of rolling resistance coefficient as the ratio between rolling resistance force and load, this leads to the following simple relation between deflection slope at $x=0$ and the SRR coefficient:

$$
C_{\mathrm{SRR}}=\frac{F_{\mathrm{SRR}}}{F_{L}}=b
$$

Using these relations on the data trace presented in Figure $4 b$, we find an SRR power of $49 \mathrm{~W} \pm 6 \mathrm{~W}$, an SRR force of $6.8 \mathrm{~N} \pm 0.8 \mathrm{~N}$, and $C_{\mathrm{SRR}}=1.4 \cdot 10^{-4} \pm$ $1.6 \cdot 10^{-5}$ or $0.014 \% \pm 0.0016 \%$.

The $C_{\mathrm{SRR}}$ values for all measurement sets were found following this procedure, and the results are presented in Figure 5. Here, the different groups are marked with different colors, the symbols represent the mean values of the three repeated measurements, and the error bars are found as the standard deviation of the three measurements. We see that the $C_{\mathrm{SRR}}$ value varies considerably over the traveled distance, from $0.005 \%$ to $0.05 \%$, with most data points in the region from $0.01 \%$ to $0.02 \%$. The method shows a good reproducibility with low standard deviations, even in regions where the $C_{\mathrm{SRR}}$ changes rapidly with distance. This demonstrates that the method is robust and can measure the $C_{\mathrm{SRR}}$ values of the road precisely, with high spatial resolution even under changing pavement conditions.

The different data groups are indicated with red, green, and blue on Figure 5. Average values of $P_{\mathrm{SRR}}$, $F_{\mathrm{SRR}}$ and $C_{\mathrm{SRR}}$ for each group are shown in Table 2. The groups were divided based on the location of the maximum, captured by Sensors 4, 5, and 6, and it is possible to see a clear difference in the SRR values within the different groups. Furthermore, the variations in $C_{\mathrm{SRR}}$, with distance seen in Figure 5 follow the trends seen in the measured deflection slopes in Figure 3. This is because a large deflection slope signal in the sensors closest to the axle (Sensors 3 and 4) generally results in a high intersection value with the $y$ - axis, and thus a high calculated $C_{\mathrm{SRR}}$ (Equation 5).

The magnitude and the location of the peaks in the deflection slope curves are determined by the shape of the deflection basin, which mainly is controlled by the relative stiffness of the top asphalt layer compared with the lower layers. For situations with a relatively stiff top layer, the deflection basin will be broad and have a small amplitude, resulting in curves like those of Group 3 and a small SRR. A relatively soft top layer, on the 


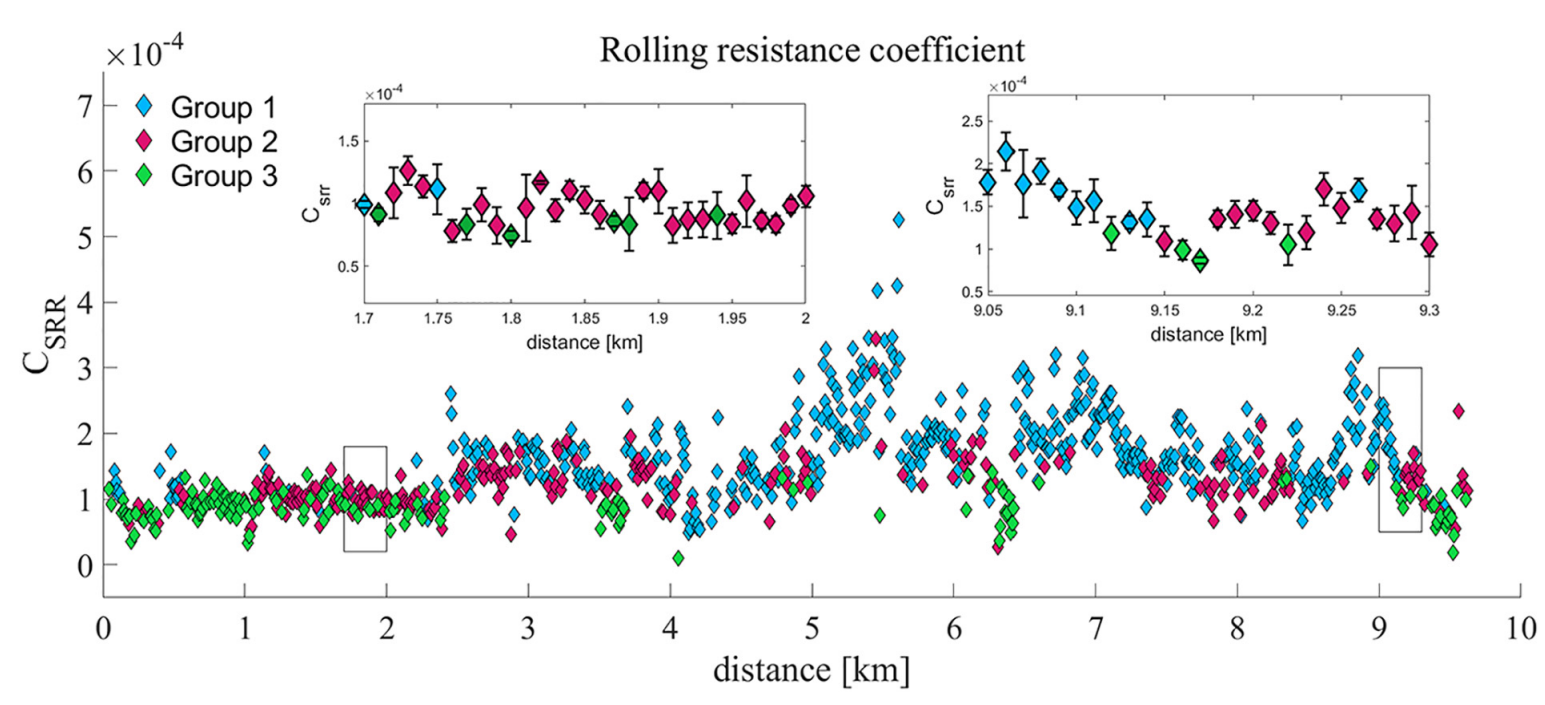

Figure 5. Calculated $C_{\mathrm{SRR}}$ values plotted versus distance, with insets showing a steady and a varying section with standard deviations illustrated by error bars. The different colors represent the three different Groups (see Table I).

Note: $C_{\mathrm{SRR}}=$ structural rolling resistance coefficient.

Table 2. Average $C_{\text {SRR }}, F_{\text {SRR }}$, and $P_{\text {SRR }}$ for the Three Groups of Traffic Speed Deflectometer Data

\begin{tabular}{|c|c|c|c|c|}
\hline Group & $C_{\text {SRR }}[-]$ & $P_{\text {SRR }}(\mathrm{W})$ & $F_{\text {SRR }}(\mathrm{N})$ & \# in group \\
\hline $\begin{array}{l}\text { Group } 1 \\
\text { Group } 2 \\
\text { Group } 3\end{array}$ & $\begin{array}{l}1.7 \cdot 10^{-4} \pm 6 \cdot 10^{-5} \\
1.2 \cdot 10^{-4} \pm 4 \cdot 10^{-5} \\
0.9 \cdot 10^{-4} \pm 3 \cdot 10^{-5}\end{array}$ & $\begin{array}{r}124.2 \pm 57 \\
84.9 \pm 30 \\
61.7 \pm 21\end{array}$ & $\begin{array}{l}8.6 \pm 3.0 \\
5.9 \pm 1.8 \\
4.2 \pm 1.3\end{array}$ & $\begin{array}{l}506 \\
272 \\
159\end{array}$ \\
\hline
\end{tabular}

Note: It can be seen that SRR for data in Group I is largest, followed by Group 2, and then Group 3. The number of measurements within the dataset belonging to each group is listed in the last column. $C_{\mathrm{SRR}}=\mathrm{SRR}$ coefficient; $F_{\mathrm{SRR}}=$ rolling resistance force; $P_{\mathrm{SRR}}=$ dissipated power due to $S R R ; S R R=$ structural rolling resistance.

other hand, will give a deep and narrow basin, giving deflection curves like those of Group 1 and a higher SRR. This is consistent with what is visible in the measurements.

\section{Impact of a Finite Contact Surface}

For the calculations of the dissipated power and $C_{\mathrm{SRR}}$ above, it is assumed that the interaction between tire and road can be described as a point load. This is a simplification of the real interaction between the tire and the pavement where the contact surface has a finite area. To investigate whether this approximation has a significant influence on the calculated SRR loss, an expression is adopted for the power dissipation derived by (9). The expression is based on a moving reference frame with constant velocity, which is consistent with the TSD setup. Furthermore, it is assumed that the tire is elastic and therefore does not dissipate energy and that the tire provides a uniform applied stress to the surface,

$$
P_{\mathrm{SRR}}^{\text {contact area }}=p v \int_{S} \frac{\partial z(X, y, z)}{\partial X} d S
$$

where

$p$ is the tire pressure,

$v$ is the driving speed,

$Z$ is the vertical component of the displacement field of the pavement surface, and

$\frac{\partial z(X, y, z)}{\partial X}$ is the deflection slope.

The integral is taken over the contact surface, $S$, which is the area where the tire is in contact with the pavement. Plugging in a linearly varying deflection slope and assuming a circular contact area obtains

$$
\begin{aligned}
P_{\mathrm{SRR}}^{\mathrm{contact} a r e a} & =p v \int_{S} \frac{\partial z(X, y, z)}{\partial X} d S \\
& =p v \int_{-r}^{r} \int_{-\sqrt{r^{2}-X^{2}}}^{\sqrt{r^{2}-X^{2}}}(a X+b) d y d X \\
& =p v b \pi r^{2}=F v b=P_{\mathrm{srr}}^{\text {point load }}
\end{aligned}
$$


Thus, for a linearly varying deflection slope the power dissipated over a finite contact area is equal to the power dissipated at a point load.

\section{Model Calculation of Pavement Response}

So far, it has been assumed that the deflection slope underneath the tire is linear and can be found by interpolation between the two sensors nearest to the axle. The validity of this assumption will now be investigated by use of simulated deflection slopes. The purpose of this is solely to generate curves with similar behaviors to those observed in the measurements, and to investigate how well the assumption of a linear deflection slope performs for the simulated curves. In particular, this is not an attempt to model the exact pavement response measured, but rather a theoretical exploration of the interpolation approach.

For simulating the pavement response, the investigation uses the time-domain based viscoelastic solver ViscoWave II-M, developed at Michigan State University $(21,22)$. ViscoWave II-M employs the socalled spectral element method to solve the wave propagation problem in the pavement structure and calculate the pavement response to an arbitrary loading. The model can simulate the time-dependent responses and allows each pavement layer to be either elastic or viscoelastic (23).

The program was modified slightly for this study such that the simulated conditions are similar to the TSD setup and therefore can be used for comparison. The original solver calculates the pavement deflection under the tire in a steady reference frame. The modified version calculates the response between the two tires in the tire reference frame, that is, a moving reference frame. From the simulated deflection curve the corresponding slope is calculated and filtered to remove numerical noise.

The pavement structure used for the simulation consists of three layers, representing an asphalt layer, a base layer, and a subgrade layer. Four different pavement models with identical construction are simulated, only changing viscoelastic parameters for the asphalt (top) layer. The parameters for the structure (height, elastic moduli, Poisson's ratio and density) are chosen to be typical values for these kinds of pavement layers and they are listed in Table 3. The viscoelastic properties of the asphalt layer are described by the relaxation modulus $E(t)$, given by

$$
\begin{gathered}
\log (E(t))=c_{1}+\frac{c_{2}}{1+e^{\left(-c_{3}-c_{4} \log \left(t_{R}\right)\right)}} \\
\log \left(t_{R}\right)=\log (t)-\log \left(a_{T}\right)
\end{gathered}
$$

where
Table 3. Mechanical Characteristics for the Simulated Pavement

\begin{tabular}{lll}
\hline Asphalt & Base & Subgrade \\
\hline$E(t)$ & $E_{2}=124.3 \mathrm{MPa}$ & $E_{3}=65.4 \mathrm{MPa}$ \\
$\nu=0.35$ & $v=0.35$ & $\nu=0.45$ \\
$\rho=2,322.7 \frac{\mathrm{kg}}{\mathrm{m}^{3}}$ & $\rho=2,082.4 \frac{\mathrm{kg}}{\mathrm{m}^{3}}$ & $\rho=1,762 \frac{\mathrm{kg}}{\mathrm{m}^{3}}$ \\
$h=0.15 \mathrm{~m}^{2}$ & $h=0.3 \mathrm{~m}$ & $h=\infty$
\end{tabular}

Note: All pavement structures are made of three layers, each characterized by their Poisson's Ratio $(v)$, mass density $(\rho)$, average thickness $(h)$ and the relaxation modulus $(E)$. The relaxation modulus for the asphalt layer is given by Equation 8 .

\begin{tabular}{|c|c|c|c|c|}
\hline \multirow[b]{2}{*}{ Properties } & \multicolumn{4}{|c|}{ Pavements } \\
\hline & PAVI & PAV2 & PAV3 & PAV4 \\
\hline \multicolumn{5}{|l|}{ Sigmoid coefficients } \\
\hline $\overrightarrow{c_{1}}$ & 1.4 & $\mathrm{I} .054$ & 0.978 & 1.67 \\
\hline$c_{2}$ & 2.04 & 2.986 & 3.8 & 3.39 \\
\hline$c_{3}$ & 0.944 & 0.335 & 0.521 & 0.981 \\
\hline$C_{4}$ & -0.417 & -0.436 & -0.519 & -0.767 \\
\hline Shift factor $\log \left(a_{T}\right)$ & 0.37 & 0.32 & 0.49 & 0.34 \\
\hline \multicolumn{5}{|l|}{$\mathrm{E}(\mathrm{t})$ characteristics } \\
\hline$E_{0}[\mathrm{MPa}]$ & 2,753 & 10,956 & 59,970 & || $4,820$ \\
\hline$E_{0}-E_{\infty}[\mathrm{MPa}]$ & 2,728 & 10,945 & 59,960 & 114,770 \\
\hline $\begin{array}{l}\text { Stiffness } \\
\text { Amount of damping }\end{array}$ & & & & \\
\hline
\end{tabular}

Table 4. Properties of the Four Different $E(t)$ Used for the Study

$c_{1}, \ldots, c_{4}$ are the sigmoid coefficients

$t_{R}$ is the reduced time, and

$a_{T}$ is the shift factor (16).

The parameters for the relaxation moduli are taken from backcalculated falling weight deflectometer tests on road segments located in California, to have realistic $E(t)$ curves (16). The characteristics of these moduli range from very stiff with high damping to very soft with little damping (see Table 4). These sets of parameters generated deflection slope curves with a similar variation to that seen in the data groups as shown in Figure $6 a$. In the simulated deflection curves, the stiff pavement with large damping (PAV4) shows a small deflection and deflection slope peaks far apart, whereas the soft pavement with little damping (PAV1) has the opposite behavior. Probably, other choices of pavement parameters could result in similar deflection basins. However, for the present purpose the detailed input parameters of the model are not so important, as long as they are reasonably realistic.

In Figure $6 b$ a zoom of the contact region for each of the simulated deflection slope curves is shown. The contact area between the tire and pavement is assumed circular with radius $(r)$ and the interval $[-r ; r]$ is marked with gray color. The idea is to determine how much the actual SRR in the simulated deflection slope curve deviates from 


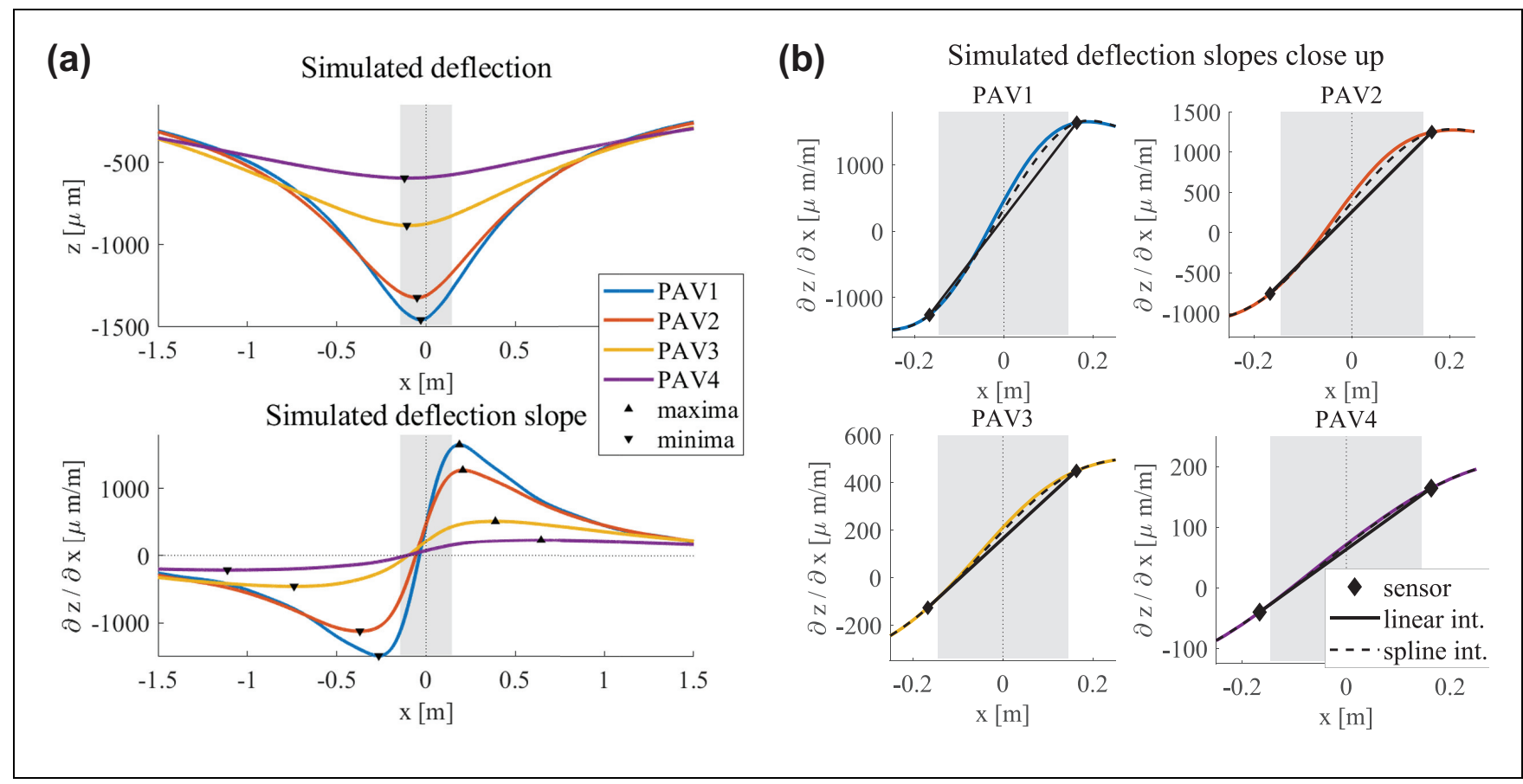

Figure 6. (a) Simulated deflection and deflection slope curves for four pavements with different $E(t)$ of the asphalt layer, and $(b)$ close up of the simulated deflection slope curves with a linear and a cubic spline interpolation. The contact area interval is marked with gray color.

the SRR obtained by assuming a linear interpolation between coordinates of the two sensors closest to the axle in the measurement. The linear interpolation is marked on Figure $6 b$ by a black line and the SRR is found as the intersection of this linear interpolation with the $z$-axis.

Calculating the SRR for the simulations involves integrating the deflection slope over the contact area as described above in Equation 6, again assuming a circular contact area with origin in $x=0$ and radius $r$. A value of $r=14.5 \mathrm{~cm}$, found from the tire pressure and axle load of the TSD, is used.

In addition to the linear interpolation, a cubic spline interpolation was also created. In this, a 3 rd order polynomial is used to find the values in between the two interpolation points instead of a linear function, thus giving a smoother interpolation curve. As this method has more unknown parameters to fit than the linear, nine simulation points are used, corresponding with the coordinates of the TSD sensors, to make the interpolation. The spline interpolation is marked on Figure $6 b$ with a dotted line. The spline interpolation is included in an attempt to approximate the actual deflection slope in the contact area better.

The relative difference between the interpolations and the simulation curves is found by the relative difference in the dissipated energy over the contact area,

$$
\frac{\Delta P^{\text {int. }}}{P}=\frac{\int_{S} \frac{\partial z^{\text {sim }}}{\partial x} d S-\int_{S} \frac{\partial z^{\text {int. }}}{\partial x} d S}{\int_{S} \frac{\partial z^{\text {im }}}{\partial x} d S}
$$

Table 5. Calculated Change in $P_{\text {SRR }}$ of the Simulated Deflection Slope and the Linear and Cubic Spline Interpolations for Different Pavements, Also Showing Values for the Calculated PSRR of Both the Simulation and the Interpolations for Each Pavement

\begin{tabular}{lcccc}
\hline Pavement & PAVI & PAV2 & PAV3 & PAV4 \\
\hline$P_{\text {srr }}^{\text {sim }}$ & $335 \mathrm{~W}$ & $356 \mathrm{~W}$ & $170 \mathrm{~W}$ & $59 \mathrm{~W}$ \\
$P_{\text {srrear }}^{\text {liner }}$ & $172 \mathrm{~W}$ & $220 \mathrm{~W}$ & $134 \mathrm{~W}$ & $54 \mathrm{~W}$ \\
$P_{\text {srr }}^{\text {spline }}$ & $257 \mathrm{~W}$ & $297 \mathrm{~W}$ & $159 \mathrm{~W}$ & $58 \mathrm{~W}$ \\
$\frac{\Delta \text { Plinear }}{P}$ & $49 \%$ & $38 \%$ & $17 \%$ & $9 \%$ \\
$\frac{\Delta P_{\text {spline }}}{P}$ & $23 \%$ & $17 \%$ & $6 \%$ & $2 \%$ \\
\hline
\end{tabular}

The calculated $P_{\mathrm{SRR}}$ values for the different deflection slope curves and the two interpolated curves are listed in Table 5 along with their relative differences.

The analysis shows that the difference between the simulated deflection slope and the linear interpolation is small for PAV4, $\frac{\Delta P^{\text {linear }}}{P}=9 \%$, where the deflection maximum and minimum are far apart. With decreasing stiffness, and thus smaller distance between maximum and minimum, the error increases, with the largest deviation found in PAV1, where $\frac{\Delta P^{\text {linear }}}{P}=49 \%$.

The spline interpolation shows the same trend, but it gives a better estimate of SRR. Thus, for the PAV1 the difference is only $\frac{\Delta P^{\text {spline }}}{P}=23 \%$, whereas for PAV4 it gives practically the same value as the model curve.

It can be concluded that the linear assumption is valid when the deflection slope peaks are far apart, whereas it 
underestimates SRR when the peaks are too close to the origin to be resolved. The spline interpolation in all cases gives a slightly better estimate of SRR, especially for pavements for which the peaks are close together.

Lastly, the numerical calculations were employed to estimate the difference in the deflection slope obtained underneath the tires and at the location of the TSD sensors. In the TSD setup, the sensors are located between the tire pair (see Figure $2 a$ ) and therefore the deflection slopes reported in this paper are measured in between the tire pair. This deviates from the analysis assumptions about the contact area in Equation 6, where it is assumed to be circular with origin in $x=0$. By simulating the pavement deflection for pavement PAV1 directly underneath the tires and in between the tire pair, respectively, it was found that the difference in $P_{\mathrm{SRR}}$ is $3.6 \%$. Consequently, this does not have a significant impact on the final SRR results.

\section{Summary and Outlook}

This paper has presented a model-free way to estimate SRR from pavement deflection slope measurements obtained with the TSD. In the simplest approach, it was assumed that the contact between tire and road is pointlike (i.e., a "moving point load"). In that case, the SRR coefficient, $C_{\mathrm{SRR}}$, is simply given as the value of the deflection slope curve at the position of the point load. Because it is not possible to measure exactly at that position because of the presence of the axle, the deflection slope was estimated from a linear interpolation of nearby measurement points behind and in front of that location. The point load assumption is shown to be equivalent to calculations based on a finite contact area, if the deflection slope varies linearly within the contact region.

A set of data from a test road was investigated and the values of $C_{\mathrm{SRR}}$ found by this method span from $0.005 \%$ to $0.05 \%$, which are modest values compared with typical tire rolling resistance coefficients that are in the range $0.5 \%$ to $1 \%$. The values are slightly lower than those found in empirical and numerical studies on the subject $(9-11,15)$. The data were divided into three groups based on how much of the deflection slope maximum was resolved by the TSD sensors. This was based on the hypothesis that this criterion is critical for the linear interpolation to be a good estimate of the deflection slope under the tire. It was found that for measurements in Group 1 with maximum located closest to the load, the SRR was highest, and for Group 3 with maximum located the furthest away, the SRR was lowest. Through simulated deflection slope curves obtained using the program ViscoWave II-M the linear interpolation was found to underestimate the actual SRR by up to $\sim 50 \%$ in the worst case. Using a cubic spline interpolation between nine positions corresponding to the TSD sensor positions improved the SRR estimate considerably, confirming that the resolution of the maximum is critical for the linear interpolation approach to give accurate results. Further development of the interpolation method will improve the method and improve the accuracy of the estimated SRR values. By use of numerical studies the authors aim to develop a simple functional expression that will allow the deflection slope values underneath the axle to be estimated with greater accuracy.

The strength of the method is that it requires no knowledge about the pavement structure or pavement properties. Furthermore, the use of the TSD vehicle makes data collection relatively fast and easy and the deflection slope measurements are very precise. This leads to reproducible values of $C_{\mathrm{SRR}}$ determined with low standard deviation, even in areas of the road where the values vary considerably.

The measurements included in this study were made on a test road with the purpose of illustrating the new method and this was chosen for purely practical reasons. They were carried out in relatively cold conditions (pavement temp. $\sim 18^{\circ} \mathrm{C}$ ) and a future study with higher pavement and air temperature is expected to provide higher SRR values. In the study, it was found that the magnitude and location of the maximum deflection slope is correlated with the SRR. It is expected that these quantities are mainly dependent on the relative stiffness of the top layer compared with the underlying layers and that the location of the maximum deflection depends on the amount of damping in the pavement (damping in top layer, foundation, or a combination). The relationship between these pavement characteristics and the behavior of the deflection slope curve should be explored further by use of simple physical models.

Through this new, easy method for measuring SRR, it will be feasible to conduct a series of tests on roads with different pavement structures and thus investigate the relationship between pavement structure and SRR. Furthermore, the impact of road temperature or driving speed could also be investigated. Such large-scale systematic surveys could provide much needed clarity in the study of SRR, and establish under which circumstances SRR is important for overall fuel consumption as well as how it is affected by various parameters.

\section{Author Contributions}

The authors confirm contribution to the paper as follows: study conception and design: Natasja R. Nielsen, Christoffer P. Nielsen, Tina Hecksher, Poul G. Hjorth; data collection: Christoffer P. Nielsen; analysis and interpretation of results: Natasja R. Nielsen, Christoffer P. Nielsen, Imen Zaabar, Karim Chatti; draft manuscript preparation: Natasja R. Nielsen, Christoffer P. Nielsen, Tina Hecksher, Poul G. Hjorth, Karim Chatti, Imen Zaabar. All authors reviewed the results and approved the final version of the manuscript. 


\section{Declaration of Conflicting Interests}

The authors declared no potential conflicts of interest with respect to the research, authorship, and/or publication of this article.

\section{Funding}

The authors received no financial support for the research, authorship, and/or publication of this article.

\section{References}

1. Chupin, O., J. M. Piau, and A. Chabot. Effect of Bituminous Pavement Structures on the Rolling Resistance. Proc., 11th International Conference on Asphalt Pavements, Nagoya, Japan, 2010, pp. 1287-1296.

2. Hall, D. E., and J. C. Moreland. Fundamentals of Rolling Resistance. Rubber Chemistry and Technology, Vol. 74, No. 3, 2001, pp. 525-539.

3. Coleri, E., and J. T. Harvey. Impact of Pavement Structural Response on Vehicle Fuel Consumption. Journal of Transportation Engineering, Part B: Pavements, Vol. 143, No. 1, 2017, p. 04017002. https://doi.org/10.1061/ JPEODX.0000004.

4. Louhghalam, A., M. Akbarian, and F. J. Ulm. Scaling Relationships of Dissipation-Induced Pavement-Vehicle Interactions. Transportation Research Record: Journal of the Transportation Research Board, 2014. 2457: 95-104.

5. Bazi, G., E. Y. Hajj, A. Ulloa-Calderon, and P. Ullidtz. Finite Element Modelling of the Rolling Resistance Due to Pavement Deformation. International Journal of Pavement Engineering, 2018, pp.1-11. https://doi.org/10.1080/ 10298436.2018.1480778.

6. Louhghalam, A., M. Akbarian, and F. J. Ulm. Scaling Relationships of Dissipation-Induced Pavement-Vehicle Interactions. Transportation Research Record: Journal of the Transportation Research Board, 2015. 2457: 95-104.

7. Flügge, W. Viscoelasticity. Springer, Berlin, Heidelberg, 1975.

8. Balzarini, D., I. Zaabar, and K. Chatti. Effect of Pavement Structural Response on Rolling Resistance and Fuel Economy using a Mechanistic Approach. Advances in Materials and Pavement Performance Prediction, Vol. 10, 2018, pp. 49-51.

9. Chupin, O., J. M. Piau, and A. Chabot. Evaluation of the Structure-Induced Rolling Resistance (SRR) for Pavements Including Viscoelastic Material Layers. Materials and Structures/Materiaux et Constructions, Vol. 46, No. 4, 2013, pp. 683-696. https://doi.org/10.1617/s11527-0129925-z.

10. Akbarian, M., S. S. Moeini-Ardakani, F. Ulm, and M. Nazzal. Mechanistic Approach to Pavement-Vehicle Interaction and Its Impact on Life-Cycle Assessment. Transportation Research Record: Journal of the Transportation Research Board, 2012. 2306: 171-179.

11. Zaabar, I., and K. Chatti. A Field Investigation of the Effect of Pavement Type on Fuel Consumption. Proc., T\&DI Congress 2011: Integrated Transportation and
Development for a Better Tomorrow, Chicago, IL, 2011, pp. 772-781.

12. Balzarini, D., I. Zaabar, and K. Chatti. Impact of Concrete Pavement Structural Response on Rolling Resistance and Vehicle Fuel Economy. Transportation Research Record: Journal of the Transportation Research Board, 2017. 2640: 84-94.

13. Harvey, J. T., J. D. Lea, C. Kim, E. Coleri, I. Zaabar, A. Louhghalam, K. Chatti, J. Buscheck, and A. But. Simulation of Cumulative Annual Impact of Pavement Structural Response on Vehicle Fuel Economy. Research Report: UCPRC-RR-2015-05. University of California Pavement Research Center, Davis, CA, 2016.

14. Louhghalam, A., M. Akbarian, and F. J. Ulm. Flügge's Conjecture: Dissipation-Versus Deflection-Induced Pavement-Vehicle Interactions. Journal of Engineering Mechanics, Vol. 140, No. 8, 2013, p. 04014053. https:// doi.org/10.1061/(asce)em.1943-7889.0000754.

15. Pouget, S., C. Sauzéat, H. D. Benedetto, and F. Olard. Viscous Energy Dissipation in Asphalt Pavement Structures and Implication for Vehicle Fuel Consumption. Journal of Materials in Civil Engineering, Vol. 24, No. 5, 2012, pp. 568-576. https://doi.org/10.1061/(ASCE)MT. 1943-5533.0000414.

16. Balzarini, D., K. Chatti, I. Zaabar, A. A. Butt, and J. T. Harvey. Mechanistic-Based Parametric Model for Predicting Rolling Resistance of Flexible Pavements. Transportation Research Record: Journal of the Transportation Research Board, 2019. 2673: 341-350.

17. Hildebrand, G., and S. Rasmussen. Development of a High Speed Deflectograph. Technical Report 117. Road Directorate, Danish Road Institute, 2002.

18. Krarup, J., S. Rasmussen, L. Aagaard, and P. G. Hjorth. Output from the Greenwood Traffic Speed Deflectometer. Proc., 22nd ARRB Conference: Research into Practice, South Vermont, Australia, 2006.

19. Chai, G., S. Manoharan, A. Golding, G. Kelly, and S. Chowdhury. Evaluation of the Traffic Speed Deflectometer Data using Simplified Deflection Model. Transportation Research Procedia, Vol. 14, 2016, pp. 3031-3039. https:// doi.org/10.1016/j.trpro.2016.05.444.

20. Nielsen, C. P. Visco-Elastic Back-Calculation of Traffic Speed Deflectometer Measurements. Transportation Research Record: Journal of the Transportation Research Board, 2019. 2673: 439-448.

21. Lee, H. S. Development of a New Solution for Viscoelastic Wave Propagation of Pavements Structures and Its Use in Dynamic Backcalculations. $\mathrm{PhD}$ thesis. Michigan State University, 2013.

22. Balzarini, D., I. Zaabar, K. Chatti, and M. Losa. Impact of Flexible Pavement Structural Response on Rolling Resistance and Vehicle Fuel Consumption. Proc., World Conference on Pavement and Asset Management, Baveno, Italy, 2017.

23. Lee, H. S., H. V. Quintus, and D. Steel. Effect of Moving Dynamic Loads on Pavement Deflections and Backcalculated Modulus. Presented at 97th Annual Meeting of the Transportation Research Board, Washington, D.C., 2018. 